\title{
Análisis del comportamiento emocional en alumnos de educación secundaria en el aprendizaje virtual
}

\section{Analysis of emotional behavior in high school students in virtual learning}

1 Juan Carlos Sarmiento Saavedra

http://orcid.org/0000-0001-8114-9410

Pontificia Universidad Católica del Ecuador Sede Esmeraldas, Maestría en Tecnología de la Información, Esmeraldas, Ecuador

juan.sarmiento@pucese.edu.ec

2 Wilson Gustavo Chango Sailema

http://orcid.org/0000-0003-3231-0153

Pontificia Universidad Católica del Ecuador Sede Esmeraldas, Escuela de Sistemas y

Computación, Esmeraldas, Ecuador

wilson.chango@pucese.edu.ec

3 José Javier Mendoza Loor

http://orcid.org/ 0000-0001-8623-872X

Universidad Técnica Luis Vargas Torres de Esmeraldas Sede La Concordia, Facultad de

Tecnologías de la Información

jose.mendoza.loor@utelvt.edu.ec

4 María Angélica Troya Loor

http://orcid.org/ 0000-0001-9794-5868

Ministerio de Educación, Distrito23D03, La Concordia, Ecuador,

angelica.troya@educacion.gob.ec

Artículo de Investigación Científica y Tecnológica

Enviado: $11 / 12 / 2021$

Revisado: $25 / 12 / 2021$

Aceptado: 02/01/2022

Publicado:07/02/2022

DOI: https://doi.org/10.33262/concienciadigital.v5i1.2063

Cítese: Sarmiento Saavedra, J. C., Chango Sailema, W. G., Mendoza Loor, J. J., \& Troya Loor, M. A. (2022). Análisis del comportamiento emocional en alumnos de educación secundaria en el aprendizaje virtual. ConcienciaDigital, 5(1), 130-152. https://doi.org/10.33262/concienciadigital.v5i1.2063

CONCIENCIA DIGITAL, es una Revista Multidisciplinar, Trimestral, que se publicará en soporte electrónico tiene como misión contribuir a la formación de profesionales competentes con visión humanística y crítica que sean capaces de exponer sus resultados investigativos y científicos en la misma medida que se promueva mediante su intervención cambios positivos en la sociedad. https://concienciadigital.org

La revista es editada por la Editorial Ciencia Digital (Editorial de prestigio registrada en la Cámara Ecuatoriana de Libro con No de Afiliación 663) www.celibro.org.ec 


Palabras
claves:
emoción,
aprendizaje
virtual,
autorregulación,
plataforma
digital.

Keywords: emotion, elearning, selfregulation, digital platform.
Resumen

Introducción. La educación evoluciona constantemente y cada vez mejora con la innovación de nuevas metodologías, estrategias, recursos y el avance tecnológico. Objetivo. Comparar el rendimiento académico de los estudiantes de educación mediante emociones para mejorar el aprendizaje virtual. Metodología. Se empleó la investigación de la ciencia del diseño. Los métodos que se aplicaron fueron el analítico, sintético y experimental. El estudio se realizó en la Unidad Educativa "Nueva Concordia", a un grupo de once estudiantes que cursan el bachillerato obteniendo un muestreo no probabilístico debido a la pandemia mundial. El instrumento que se aplicó en la encuesta fue un cuestionario, que mediante la escala de Likert permitió medir la valoración de las emociones del estudiante y para el índice de consistencia interna se empleó el coeficiente Alfa de Cronbach con un valor de 0,82 de muy alta confiabilidad y validez. Luego para indicar el grado de relación estadística entre las emociones y las notas se aplicó el coeficiente de correlación de Pearson. Resultados. Los resultados mostraron que las emociones; felicidad, sorpresa y asco están correlacionadas positivamente y las emociones miedo y neutral de forma negativa con la nota del tutor virtual. Conclusión. La investigación permite concluir que las emociones no están fuertemente correlacionadas con la nota del estudiante luego de la interacción, sin embargo, sí influyen en la misma. Esto se debe a que todas las personas son distintas y aunque se encuentren algunas similitudes en este sentido no quiere decir que se cumplan de forma generalizada en todas las personas.

\section{Abstract}

Introduction. Education is constantly evolving and improving with the innovation of new methodologies, strategies, resources and technological advances. Objective. To compare the academic performance of education students through emotions to improve virtual learning. Methodology. Design science research was used. The methods applied were analytical, synthetic and experimental. The study was carried out in the "Nueva Concordia" Educational Unit, to a group of eleven high school students, obtaining a nonprobabilistic sampling due to the world pandemic. The instrument applied in the survey was a questionnaire, which by means of the Likert scale allowed measuring the valuation of the student's 
emotions and for the internal consistency index the Cronbach's Alpha coefficient was used with a value of 0.82 of very high reliability and validity. Then, to indicate the degree of statistical relationship between emotions and grades, Pearson's correlation coefficient was applied. Results. The results showed that the emotions; happiness, surprise and disgust are positively correlated and the emotions fear and neutral negatively correlated with the virtual tutor's grade. Conclusion. The research allows us to conclude that emotions are not strongly correlated with the student's grade after the interaction, however they do influence it. This is due to the fact that all people are different and although some similarities are found in this sense it does not mean that they are fulfilled in a generalized way in all people.

\section{Introducción}

Los educadores con el paso del tiempo han estado intrigados con el alcance que tiene la tecnología para ayudar a evolucionar la educación y optimizar el aprendizaje del estudiante (Franco, 2017). La educación prospera constantemente y cada vez debe ir innovándose con nuevas metodologías, estrategias, recursos, entre otros (Estrada \& Boude, 2015). En este sentido, al hacerlo sugiere que la línea de la educación virtual tiene una relación directa con las herramientas tecnológicas que se aplican para generar contenidos digitales, plataformas de aprendizaje virtual, mecanismos de gamificación del aprendizaje, entre otros (Espinoza \& Ricaldi, 2018). Esta comprensión es crucial porque las emociones son reacciones de movimiento o impulso que experimenta un individuo que se adapta a una situación respondiendo a algunos estímulos cuando percibimos un objeto, una persona o entornos en que se encuentre ya que son aspectos fundamentales que pueden ser manipulados para influir positivamente en el propósito en línea (Kumar \& Wan, 2019; Nikopoulou et al., 2018).

De este modo, las emociones tienen una función informativa y pueden ser utilizadas por los estudiantes para regular el aprendizaje (Molinari et al., 2016). La inteligencia emocional consiste en la destreza que tiene y despliega el individuo para controlar sus emociones y sentimientos como el de los demás (Ramachandiran et al., 2019). La implicación clave que se extrae de esto es que permite discernir la información para adiestrar sus ideas (Ángel \& Retana, 2012), sobre todo en un contexto de enseñanza presencial o virtual instaurado por el profesor con el propósito de favorecer al progreso de capacidades y destrezas en sus educandos (Estrada \& Boude, 2015). 
El sistema educativo en el Ecuador ha sufrido transformaciones como los estándares de calidad, infraestructura educativa, modelo de educación y un nuevo ajuste curricular para solucionar los problemas de aprendizaje debido a que la calidad de la educación se evalúa y se da a través de una serie de indicadores entre los cuales se establece el abandono prematuro de los estudios, muchas veces por el comportamiento emocional que tienen los estudiantes al padecer de diferentes situaciones familiares en sus hogares (Barrera et al., 2017; Franco, 2017; Madrid, 2019). Sus investigaciones dejan claro que las instituciones educativas presentan una realidad distinta ya que las emociones en el aprendizaje virtual están relacionadas y contenidas en comentarios que transmiten sentimientos positivos o negativos (Clarizia et al., 2018). Algunas dificultades en los alumnos pueden ser una incorrecta formación académica en el ámbito de cómo influye el comportamiento emocional en estudiantes de secundaria en el aprendizaje virtual, por esta razón es fundamental insertar el proceso de la capacidad emocional en la malla curricular (Cifuentes, 2013).

Sin duda, el análisis de la relación entre las emociones y el aprendizaje virtual tienen un impacto significativo en la colectividad debido a la virtud de los aprendizajes por parte de los alumnos con el profesionalismo de docentes y directivos del colegio "Nueva Concordia”. La investigación es factible porque tiene el apoyo y acceso a la información verídica de la comunidad educativa ya que los beneficiarios son los padres de familia, docentes, autoridades y estudiantes, considerando que tradicionalmente demostraban el éxito o el fracaso escolar en base a la capacidad intelectual y a los buenos o malos resultados académicos ya que influyen aspectos emocionales y sociales (Greene \& Azevedo, 2010). El objetivo de la investigación es comparar el rendimiento académico de los estudiantes mediante emociones para mejorar el aprendizaje virtual. De esta manera se determina si existe alguna relación entre las emociones de los estudiantes con la nota agregada en el transcurso del aprendizaje virtual (Chango et al., 2019).

\section{Marco teórico}

En este apartado se despliega un conjunto de definiciones y tecnologías para el desenvolvimiento de la investigación, entre ellos están: la inteligencia, las emociones y el aprendizaje virtual.

\section{Inteligencia y emoción}

Los primeros acercamientos al concepto de inteligencia nacieron en la antigua Grecia. Así, por ejemplo, algunos de sus filósofos más relevantes como Platón y Aristóteles ya encontraban diferencias entre lo que ellos consideraban fenómenos internos como el pensamiento, la meditación y las pasiones o emociones (Carretero, 2015). De esta manera la inteligencia emocional se basa en los rasgos de personalidad, capacidades sociales y emocionales principalmente para el triunfo en la vida personal y competitiva del sujeto 
(León, 2009). Por lo tanto, ni el aprovechamiento académico ni el grado cognitivo determina la inteligencia emocional, por tal razón no existe ningún problema al tomarla para este trabajo.

No obstante, el rendimiento académico se conceptualiza como el nivel de estudio que adquiere el alumno según sus destrezas y habilidades hacia cada asignatura, su aprovechamiento puede ser bueno o malo debido a que se encuentra ligado a una evaluación, expresada en una nota cuantitativa o cualitativa con la intención de verificar si se ha alcanzado las metas pedagógicas anteriormente señalados y que garantizan una comprensión determinada (López, 2008).

Sin duda, la vinculación de la Inteligencia Emocional (IE) y el Rendimiento Académico (RA) ha sido un argumento de atracción para los pedagógicos, Cifuentes (2013) menciona la solidez de tratar las propias emociones reconociendo los sentimientos y solucionar inconvenientes de ambiente particular e interpersonal para la superación académica. En la figura 1, se muestra el componente interpersonal como son la empatía, relaciones interpersonales y responsabilidad social.

\section{Figura 1}

\section{Componente interpersonal y responsabilidad social}

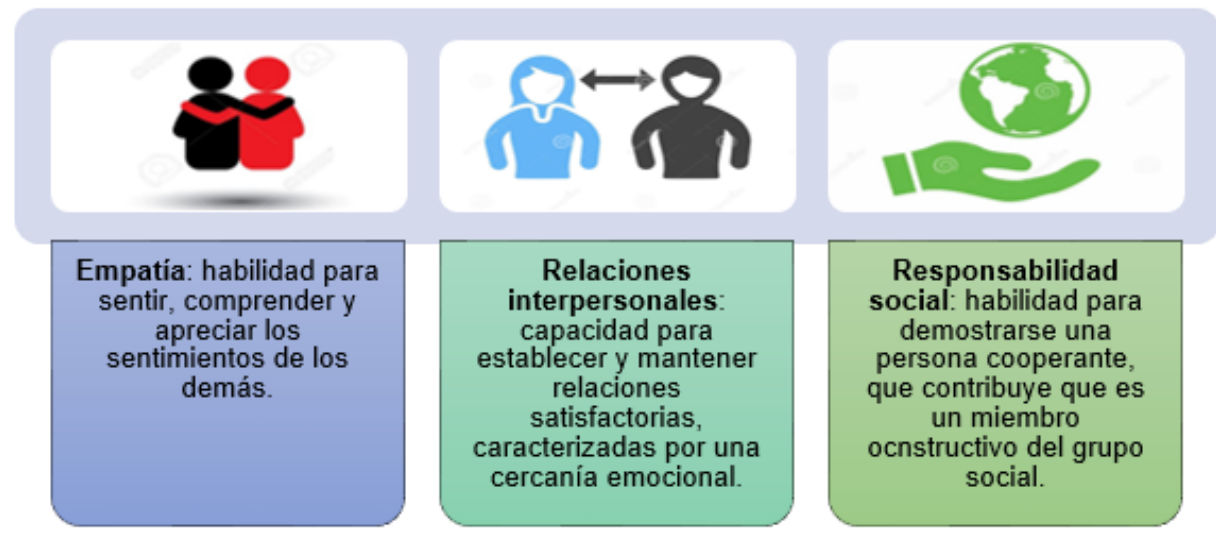

\section{Aprendizaje Virtual}

Dentro de este marco cabe señalar que existe una teoría de aprendizaje, que se desenvuelve cuando el individuo está en contacto con su contorno sociocultural y natural, lo mismo es cierto para la educación en línea (Picciano, 2013). Es indiscutible, que los educadores dependen cada vez más de los entornos de aprendizaje basados en la computadora (CBLEs) como herramientas pedagógicas, incluidos el hipertexto, los sistemas de tutoría inteligente (ITS), los mundos virtuales, las simulaciones y otros entornos que utilizan algún tipo de tecnología (Greene \& Azevedo, 2010). 
Las herramientas tecnológicas han innovado en muchos ambientes de la sociedad especialmente en el campo pedagógico cambiando de esta forma las funciones del docente siendo instructor, moderador y evaluador de los procesos de enseñanza convirtiéndolo en un tutor virtual innovador, desempeñando roles de consultor de información (Franco, 2017). En este espacio el docente permite que el estudiante sea más responsable y autónomo de su propio aprendizaje, así puede analizar, dialogar y retroalimentarse de los compañeros a través de enlaces virtuales y discusiones desde el hogar, el trabajo, entre otros (Espinoza \& Ricaldi, 2018).

En relación con este tema se logra deducir que un Entorno Virtual de Aprendizaje (EVA), proporciona las estructuras y recursos básicos en los que se realizan las interacciones de aprendizaje de los participantes, registra los eventos que ocurren durante el proceso y proporciona a los tutores herramientas básicas para analizar el aprendizaje de los estudiantes (Bogarín et al., 2017). El diseño básico que se muestra en la Figura 2 constituye las características de un EVA.

Figura 2

Diseño básico de las características de un EVA

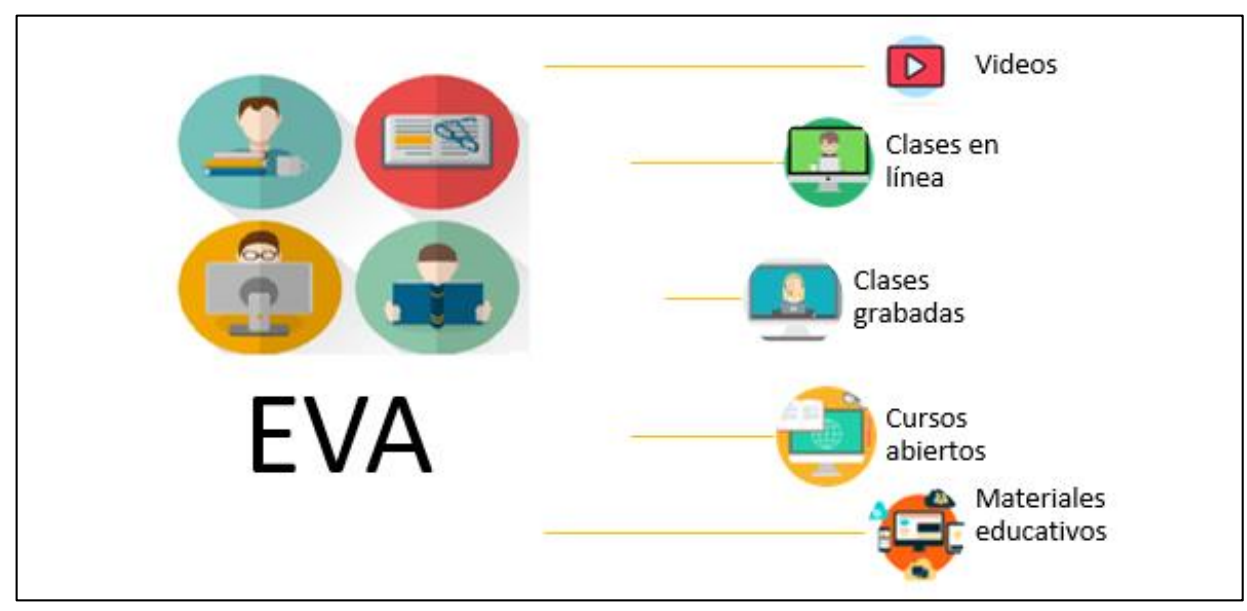

Sin embargo, las teorías de aprendizaje para la educación virtual han evolucionado en aprendizaje colaborativo en línea (ACO) con una teoría que se centra en las instalaciones del internet para proporcionar entornos de aprendizaje que fomenten la colaboración y la creación de conocimientos (Picciano, 2013). Su beneficio es de trasladar la enseñanza y el aprendizaje al internet y a la educación en red a gran escala, como en el caso de estudio del curso abierto masivo en línea de tecnología de la información y las comunicaciones (ICTMOOC) destinado a desarrollar aptitudes digitales en la cual los estudiantes interactúan entre sí (Engeness et al., 2019), esto favorece a mejorar su capacidad de aprendizaje. 
En este sentido se comprende que los educadores continuamente son atraídos por el alcance de la tecnología para ayudar a innovar el aprendizaje y optimizar la formación del alumno (Hew \& Brush, 2007). El curso abierto masivo en línea (MOOC) son la última iniciativa del aprendizaje virtual que ha dado paso a una nueva revolución en la que pueden utilizar métodos educativos de minería para la investigación de los comportamientos de aprendizaje de los estudiantes en un MOOC de informática y de esta manera mejorar la enseñanza y el aprendizaje de la experiencia en línea (Zhong et al., 2017).

Por otra parte, en la actualidad sobre el reconocimiento facial la mayoría de las investigaciones en este campo se han orientado en el estudio de rostros para explorar emociones. Las tres fases primordiales son: identificar el rostro, extraer los atributos (como eventos), e identificar la emoción en base a crónicas (Cordero \& Aguilar, 2016). Así mismo considerar las grabaciones de vídeo de las expresiones faciales y los movimientos del cuerpo para proporcionar un apoyo afectivo personalizado en un contexto educativo desde un enfoque de detección de emociones (Saneiro et al., 2015). En este mismo orden de ideas, los datos de las emociones están recopilados a partir de la interacción con entidades inteligentes, el uso de aprendizaje automático, algoritmos, multiagente computarizado para el entorno de aprendizaje (Harley et al., 2015).

Finalmente, estos fragmentos muestran los últimos avances en inteligencia artificial en un enfoque de reconocimiento de emociones en tiempo real basado en características de expresiones faciales 2D y 3D capturadas por sensores Kinect, en el cual se combina los rasgos de las unidades de animación (UA) y las posiciones de los puntos de los rasgos (FPP) rastreados por Kinect para reconocer las emociones con estos rasgos de expresión facial y un video en tiempo real (Mao et al., 2015).

\section{Trabajos relacionados}

Desde hace muchos años se ha intentado detectar los sentimientos en los rostros de las personas, el primero que trabajó en el área del reconocimiento facial fue Woodrow Wilson Bledsoe, en la década de los 60 que desarrolló un sistema llamado RAND (Dispositivo gráfico de entrada de computadora) (Bledsoe, 1963). En este campo Cerezo et al. (2020) estudia la minería de procesos para la evaluación del aprendizaje autorregulado en el aprendizaje virtual valorando los procesos y habilidades de los estudiantes que se refiere a regular las emociones, cogniciones, comportamientos y aspectos del contexto (Zimmerman, 1990), durante un curso de aprendizaje electrónico utilizando técnicas de minería de procesos empleó un nuevo algoritmo en el dominio educativo llamado Inductive Miner, mejorando el comportamiento del aprendizaje autorregulado. 
Khalfallah \& Ben Hadj Slama (2019) han investigado el efecto del análisis emocional en la mejora de los sistemas experimentales de aprendizaje electrónico, el cual consiste en proporcionar un aprendizaje más diverso, además de cursos en la web y aulas virtuales incluye a laboratorios remotos con un sistema de reconocimiento afectivo, construyendo un nuevo sistema tutorial inteligente, llamado "LabTutor" adaptada al perfil de cada estudiante. En esta línea Hew \& Cheung (2014) hacen referencia que la literatura publicada se centra en el uso del curso abierto masivo en línea (MOOCs) por instructores o estudiantes, aumentando el eficiencia de una colectividad y permiten innovar a los docentes con la didáctica de la instrucción de cursos en línea a una multitud de educandos. $\mathrm{Al}$ mismo tiempo perfeccionan diversas destrezas para mejorar los cursos.

Del mismo modo Chango et al. (2019) describen cómo aplicar la fusión de datos y preprocesamiento para crear un conjunto de datos de resumen en formato numérico y categórico por la herramienta de minería de datos Weka para seleccionar el mejor algoritmo. Continuando con las ideas, han desarrollado de manera similar un modelo teórico que une las emociones, el aprendizaje auto-regulado, y la motivación para el logro académico (Mega \& De Beni, 2014), compuesto de 3 cuestionarios de autoinforme: el test de autorregulación del aprendizaje (LQ), el test de cociente emocional (EQ), y el test de motivación (MQ).

\section{Metodología}

La investigación se efectuó en la jurisdicción de Santo Domingo de los Tsáchilas, cantón La Concordia, en la Unidad Educativa "Nueva Concordia", en el periodo lectivo (2020 2021) a un grupo de once estudiantes del bachillerato que son una muestra del cual se recogen los datos. La selección fue obtenida por un muestreo no probabilístico para los criterios considerados en la investigación se aplicó una estrategia de estratificación en la cual se eligió a estudiantes que cuentan con los recursos tecnológicos y acceso al internet debido a la pandemia mundial.

Se empleó la metodología de investigación de la ciencia del diseño con sus siglas en inglés (DSR), la cual es una nueva visión en el campo de los Sistemas de Información y Ciencias de la Computación (Bisandu, 2016), cuya finalidad según Dresch et al. (2018), es crear y evaluar artefactos de tecnología de la información destinados a resolver problemas identificados en una organización en forma rigurosa y sistemática para comunicar sus resultados. En tal sentido se empleó una exploración profunda de la literatura concerniente al tema de estudio. De igual forma con la metodología descriptiva se recopiló información de manera independiente de los alumnos al determinar sus expresiones del rostro de los videos grabados. En la metodología aplicada la información es examinada y se comparan las notas con las emociones y en la transversal se observan los cambios afectivos de los educandos en la conectividad en el proceso de la grabación de video en un momento concreto. 
Los métodos de análisis científicos que se aplicaron en la investigación fueron el analítico, sintético y experimental. El método analítico se utilizó para ejecutar un estudio de la conducta afectiva de los escolares en la instrucción en línea. Sintético puesto que del análisis se seleccionó una aplicación para identificar expresiones emocionales y por último experimental, en el que se manipuló intencionalmente la variable independiente es decir las notas y la variable dependiente que hace referencia a las emociones (Hernandez et al., 2010).

Con respecto a la técnica de investigación se aplicó la encuesta, la cual recolectó información sobre aspectos estructurales y se plantearon una serie de ítem o preguntas (Sautu, 2005), puesto que determinó como el estudiante autoevalúa sus emociones justo después de interactuar con el tutor virtual. En consecuencia, como instrumento se empleó un cuestionario, enmarcado al objetivo del trabajo autoevaluando emociones del alumnado al interactuar con el tutor virtual, de preguntas cerradas y estructuradas con opciones de respuesta única y la categoría de las interrogantes fueron en escala de Likert. Con el objeto de evaluar la fiabilidad en las escalas y el índice de consistencia interna se utilizó el alfa de Cronbach (Taber, 2018). La recolección de los datos fue en línea mediante la plataforma electrónica google forms en el siguiente enlace (https://forms.gle/NyQgDRD17LNaDaDw5)

En el procesamiento de los datos recolectados se utilizó una herramienta de análisis de emociones en videos (AEV), la cual se ejecuta bajo una versión de java, para extraer las emociones de un estudiante que se muestra en un video el cual contiene el rostro del sujeto durante la mayor parte del tiempo posible en la interacción. Como resultado del análisis la aplicación devuelve al usuario el tiempo que experimentó cada una de las emociones disponibles, la cuales son: ira, desprecio, asco, miedo, felicidad, neutral, tristeza y sorpresa. Para utilizar la aplicación fue necesario una suscripción de Api Face de Microsoft Azure y activación de una cuenta gratuita mediante un correo electrónico, obteniendo así una clave para introducir al crear un usuario de aplicación. Además, se puede visualizar a continuación un diagrama general de la arquitectura del software (AEV) en la Figura 3. 


\section{Figura 3}

Diagrama de la arquitectura del software

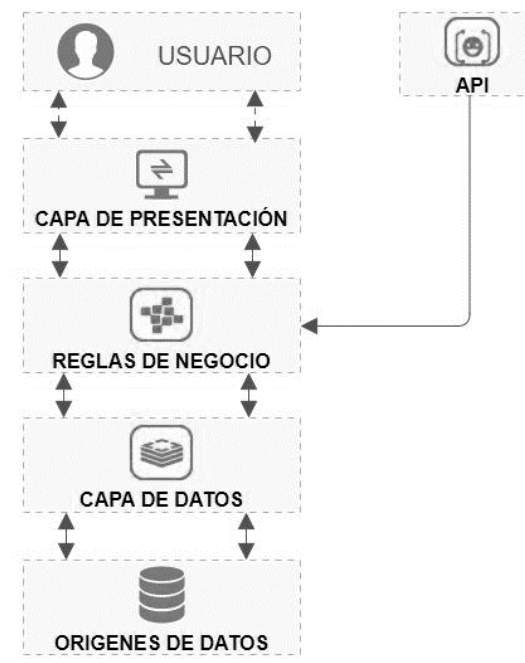

Cuando la aplicación está instalada y ejecutada se almacenan los datos del usuario y se configuran las opciones del menú de análisis, después que el usuario acepte se visualiza el menú principal y procede a seleccionar el archivo del video grabado al estudiante, el cuál descompone en fragmentos de imágenes, de esta forma se detectaron y analizaron las emociones arrojando además los resultados del individuo en porcentajes, luego se empleó una encuesta basada en las emociones que siente el estudiante en ese momento.

Posteriormente con los resultados obtenidos de las emociones y las notas de los estudiantes que se representan como variables cuantitativas continuas debido a que pueden tomar cualquier valor dentro de un intervalo (Salazar \& Castillo, 2018), luego se aplicó el test del coeficiente de correlación de Pearson el cual revela el nivel de relación estadística entre las emociones y las notas (Awofala et al., 2019), después de la interacción en el aprendizaje virtual. De esta manera, el procesamiento y análisis de las emociones queda definido como se presenta en la Figura 44.

\section{Figura 4}

Procesamiento y análisis de las emociones
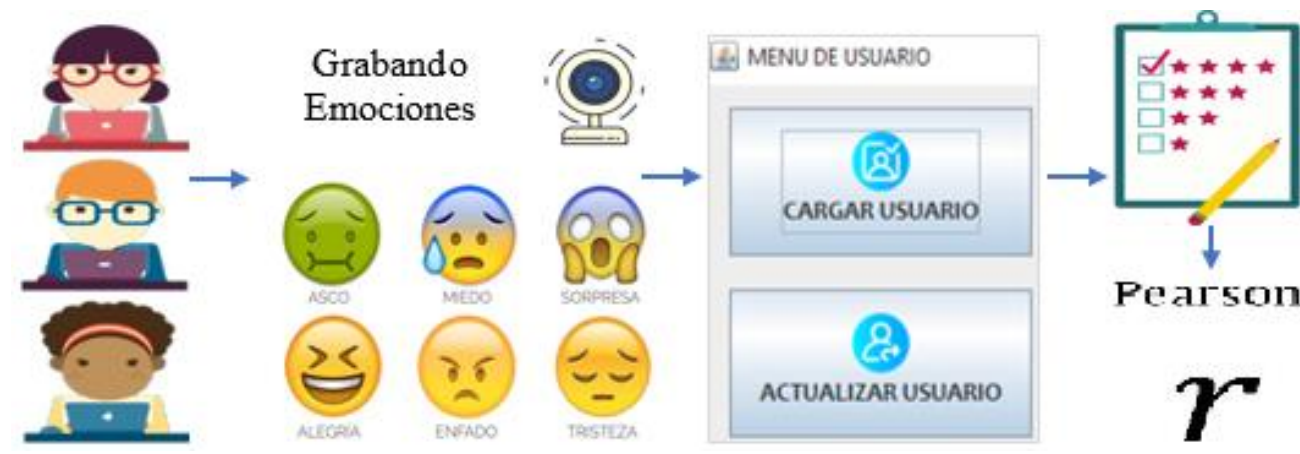
Cabe considerar que en el coeficiente de correlación de Pearson los números varían en los límites de +1 y -1 y expresan el nivel de relación entre las variables, cuando el valor de $r=0$ demuestra la inexistencia de asociación entre las variables; así mismo los valores de 1 son indicadores de una relación fuertemente positiva o negativa (Hernández et al., 2018), conforme a la escala de interpretación que se presenta en la siguiente tTabla 1.

Tabla 1

Escala de interpretación del coeficiente de correlación de Pearson

\begin{tabular}{cl}
\hline Rango de valores $r_{x y}$ & Interpretación \\
\hline $0,00 \leq r_{x y}<0,10$ & Correlación nula \\
$0,10 \leq r_{x y}<0,30$ & Correlación débil \\
$0,30 \leq r_{x y}<0,50$ & Correlación moderada \\
$0,50 \leq r_{x y}<1,00$ & Correlación fuerte
\end{tabular}

Fuente: Hernández et al. (2018)

Normas éticas

El trabajo investigativo se encuentra en los márgenes éticos y morales imperantes en la colectividad. Sin embargo, cada encuestado fue informado del motivo de la encuesta y se pidió la aprobación del representante legal antes de enviarle las interrogantes del cuestionario, descartando alguna gestión fraudulenta (Taruchaín et al., 2020).

\section{Resultados}

En esta sección desde el enfoque metodológico y a partir de los instrumentos de recolección y análisis de datos se presentan a continuación los resultados del experimento. Por lo consiguiente el experimento consistió en reunir a una serie de alumnos que estuvieron dispuestos a ser grabados (de tal forma que se muestre el rostro de la persona durante el mayor tiempo posible) mientras participaron en una sesión de interacción con un sistema de aprendizaje con un tutor virtual.

Una vez realizada la primera parte del experimento y habiendo obtenido todo el material necesario, es decir: las tabulaciones de las encuestas realizadas a los estudiantes se aplicó el coeficiente alfa de cronbach que sirvió para medir la validez y fiabilidad de la escala utilizada obteniendo un valor de 0,82 que denota una confiabilidad muy alta, tal como se muestra en la Tabla 2 de Excel donde se expresan las varianzas y los resultados. Así mismo, los vídeos de los rostros de los alumnos (a los que llamaremos sujetos) mientras están interactuando con el tutor virtual. Luego se pasó a la segunda parte de este experimento que fue analizar los vídeos, de tal forma que extrajo las emociones que ha 
sufrido el sujeto y cuánto tiempo ha poseído cada una de las emociones, en el siguiente enlace se puede observar el datasetUnico en Excel que se utilizó de los videos analizados

(drive.google.com/file/d/1R8d4v3LzOC0hwA_Z-

27fNcQaNW0J4_YN/view?usp=sharing). Por tal motivo mediante un test de correlación de Pearson se respondió a ciertas preguntas planteadas inicialmente con la relación existente entre la posesión o no posesión de un sentimiento y la nota que atribuye el tutor virtual al sujeto tras su interacción con el mismo.

\section{Tabla 2}

Tabulación de encuestas y coeficiente del Alfa de Cronbach

\begin{tabular}{|c|c|c|c|c|c|c|c|c|c|c|c|c|c|c|c|c|c|c|}
\hline \multirow[t]{2}{*}{ En } & \multicolumn{17}{|c|}{ ITEMS } & \multirow[t]{2}{*}{$\sum$} \\
\hline & 1 & 2 & 3 & 4 & 5 & 6 & 7 & 8 & 9 & 10 & 11 & 12 & 13 & 14 & 15 & 16 & 17 & \\
\hline E1 & 5 & 3 & 3 & 5 & 5 & 3 & 3 & 5 & 4 & 4 & 3 & 5 & 3 & 5 & 5 & 5 & 4 & 70 \\
\hline E2 & 5 & 4 & 4 & 3 & 4 & 3 & 5 & 3 & 4 & 4 & 2 & 2 & 3 & 5 & 3 & 5 & 3 & 62 \\
\hline E3 & 3 & 5 & 5 & 3 & 5 & 3 & 5 & 3 & 5 & 5 & 3 & 3 & 5 & 5 & 3 & 5 & 5 & 71 \\
\hline E4 & 2 & 5 & 5 & 3 & 3 & 4 & 5 & 3 & 5 & 4 & 3 & 3 & 4 & 5 & 3 & 3 & 5 & 65 \\
\hline E5 & 5 & 3 & 5 & 2 & 5 & 4 & 5 & 5 & 5 & 5 & 3 & 5 & 5 & 3 & 5 & 3 & 5 & 73 \\
\hline E6 & 5 & 5 & 3 & 4 & 5 & 4 & 5 & 4 & 3 & 5 & 5 & 5 & 4 & 5 & 2 & 5 & 5 & 74 \\
\hline E7 & 4 & 4 & 3 & 4 & 3 & 4 & 5 & 4 & 2 & 4 & 3 & 4 & 5 & 4 & 4 & 5 & 4 & 66 \\
\hline E8 & 1 & 3 & 5 & 3 & 3 & 1 & 3 & 1 & 3 & 2 & 3 & 1 & 3 & 3 & 4 & 3 & 3 & 45 \\
\hline E9 & 4 & 5 & 3 & 5 & 5 & 5 & 5 & 4 & 3 & 4 & 2 & 4 & 5 & 5 & 3 & 4 & 5 & 71 \\
\hline E10 & 5 & 3 & 5 & 5 & 5 & 3 & 5 & 4 & 3 & 4 & 3 & 4 & 4 & 5 & 4 & 3 & 5 & 70 \\
\hline E11 & 5 & 5 & 5 & 4 & 5 & 4 & 5 & 5 & 3 & 5 & 4 & 5 & 2 & 4 & 4 & 5 & 4 & 74 \\
\hline Var & 1,8 & 0,8 & 0,9 & 0,9 & 0,8 & 1 & 0,6 & 1,3 & 1 & 0,7 & 0,6 & 1,7 & 1 & 0,6 & 0,8 & 0,9 & 0,6 & \\
\hline \multicolumn{4}{|c|}{$\sum$ de varianzas } & \multicolumn{3}{|c|}{15,851} & \multicolumn{7}{|c|}{ Varianza de la suma de ítems } & \multicolumn{3}{|c|}{63,322} & \multicolumn{2}{|c|}{$\alpha: 0,82$} \\
\hline
\end{tabular}

Dentro de este orden de ideas en la Figura $\mathbf{5 5}$ se muestran varias capturas del funcionando de la herramienta análisis de emociones en videos (AEV), aplicada a cada uno de los vídeos analizados. En la ventana después de analizar se puede acceder a las opciones de resultados y gráficas estadísticas de los fotogramas, en este mismo orden en la ventana de gráficas puede acceder a cualquier emoción de sujeto analizado en forma de barras o pastel y en la ventana de resultados finales muestra un contador de cada una de las emociones, estas se pueden visualizar en porcentajes y añadir a un dataset único. 


\section{Figura 5}

Ventanas del aplicativo AEV

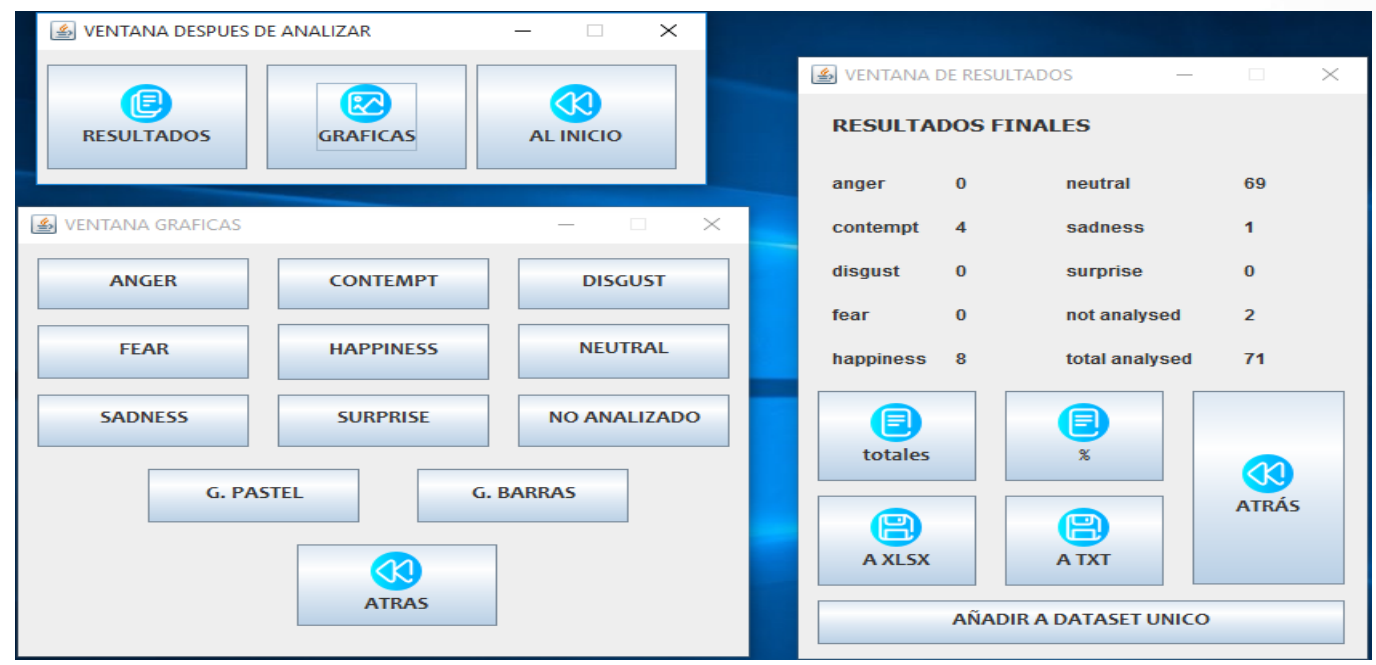

En la siguiente Figura 66 se visualiza un gráfico estadístico de sectores sobre los resultados del sujeto 5 y sus emociones en fotogramas.

\section{Figura 6}

Fotogramas de las emociones analizadas del sujeto 5

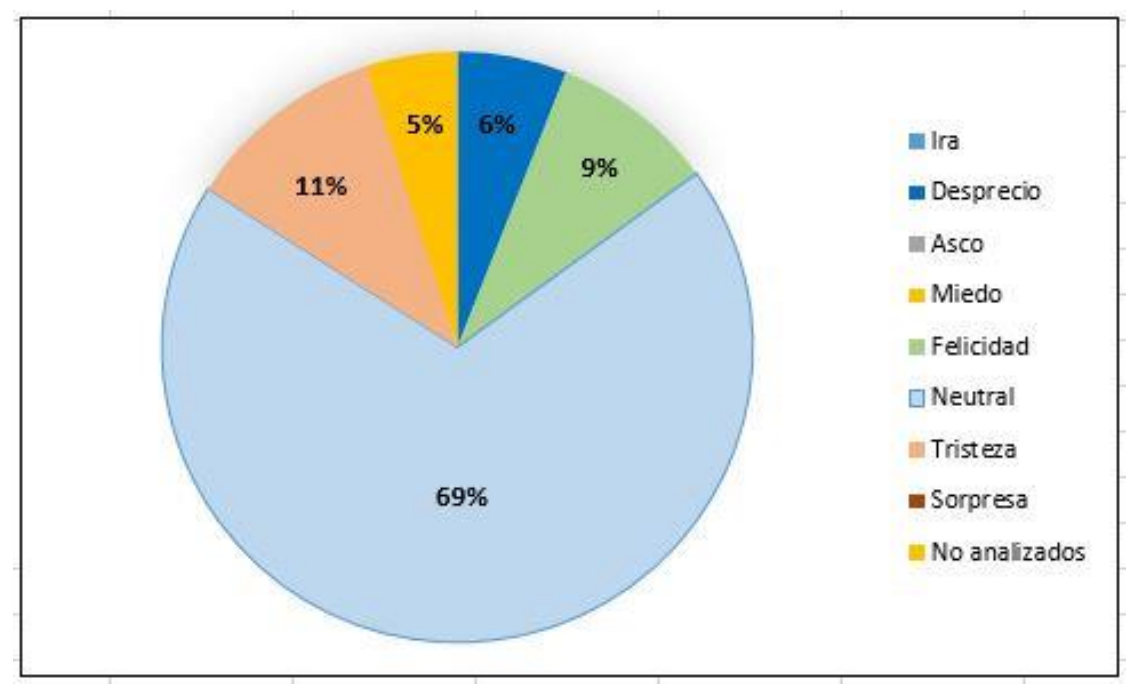

Según los datos del gráfico se puede sustentar que la emoción neutral abarca el 69\% esto es evidente tratándose de un proceso de aprendizaje educativo, por lo tanto, la variable neutral a simple vista no aporta mucha información contundente en la investigación. Sin embargo, las tres emociones que muestra el sujeto 5 como: desprecio, felicidad, tristeza y una parte de fotogramas no analizados son importantes en el análisis y la interacción. 
En la Tabla 3 se visualizan los resultados de manera total, es decir el número de fotogramas que se han analizado en los videos (totales), número de fotogramas que no se han podido analizar (no analizados) y número de fotogramas que pertenecen a cada una de las emociones disponibles.

Tabla 3

Resultados totales del análisis

\begin{tabular}{ccccccccccc}
\hline Sujeto & Ira & Desprecio & Asco & Miedo & Felicidad & Neutral & Tristeza & Sorpresa & No analizadas & Total \\
\hline S01 & 1 & 0 & 0 & 0 & 0 & 52 & 0 & 1 & 0 & 52 \\
S02 & 0 & 0 & 0 & 1 & 3 & 65 & 1 & 0 & 0 & 65 \\
S03 & 1 & 9 & 0 & 1 & 1 & 48 & 5 & 0 & 0 & 48 \\
S04 & 1 & 0 & 0 & 1 & 1 & 28 & 9 & 0 & 0 & 28 \\
S05 & 0 & 1 & 0 & 0 & 4 & 44 & 24 & 0 & 0 & 46 \\
S08 & 0 & 5 & 1 & 0 & 7 & 59 & 9 & 1 & 4 & 62 \\
S09 & 0 & 0 & 0 & 1 & 0 & 43 & 22 & 0 & 0 & 43 \\
S11 & 0 & 4 & 1 & 0 & 8 & 69 & 1 & 1 & 2 & 69 \\
\hline
\end{tabular}

Cabe mencionar que en la Tabla 3 se analizaron 11 videos en su totalidad de los cuales solo se consideraron 8 videos esto se debe a que tres de ellos arrojaron datos nulos, por tal motivo se consiguió abarcar una gran diversidad en cuanto a tipos de alumnos se refiere para así conseguir una buena muestra que se adapte lo mejor posible a la realidad. De esta misma forma en la Tabla 4 se muestran los resultados del experimento en porcentajes.

\section{Tabla 4}

Resultados en porcentajes de los fotogramas

\begin{tabular}{cccccccccc}
\hline Sujeto & \% Ira & \% Desprecio & \% Asco & \%Miedo & \%Felicidad & \% Neutral & \% Tristeza & \%Sorpresa & Útiles \\
\hline S01 & 1,23 & 0 & 0 & 0 & 0 & 100 & 0 & 1,29 & 52 \\
S02 & 0 & 0 & 0 & 1,12 & 4,62 & 100 & 1,54 & 0 & 65 \\
S03 & 1,07 & 18,75 & 0 & 1,09 & 2,08 & 100 & 10,42 & 0 & 48 \\
S04 & 1,41 & 0 & 0 & 1,21 & 3,57 & 100 & 32,14 & 0 & 28 \\
S05 & 0 & 2,17 & 0 & 0 & 8,7 & 95,65 & 52,17 & 0 & 46 \\
S08 & 0 & 8,06 & 1,36 & 0 & 11,29 & 95,16 & 14,52 & 1,37 & 62 \\
S09 & 0 & 0 & 0 & 1,04 & 0 & 100 & 51,16 & 0 & 43 \\
S11 & 0 & 5,8 & 1,44 & 0 & 11,59 & 100 & 1,45 & 1,19 & 69 \\
\hline
\end{tabular}

Respecto a los resultados de los fotogramas de esta tabla no se muestran los datos no analizados. No obstante, la columna de datos útiles son los fotogramas que se han podido examinar. Además, mostrar los resultados en porcentajes es una medida adecuada para 
que el análisis no se vea afectado por diferencias de tiempo en las sesiones. A continuación se logra visualizar en la Tabla 5 la nota que le atribuye el tutor virtual a cada uno de los sujetos luego de la interacción, calculada entre 0 a 10 fundamentada en el Art. 194 de la escala de calificaciones del reglamento general de la LOEI (Asamblea Nacional, 2015).

\section{Tabla 5}

Notas de los sujetos

\begin{tabular}{cc}
\hline Sujeto & Nota \\
\hline S01 & 8 \\
S02 & 7 \\
S03 & 6 \\
S04 & 8 \\
S05 & 9 \\
S08 & 10 \\
S09 & 4 \\
S11 & 6 \\
\hline
\end{tabular}

Entorno a este aspecto del experimento se ha realizado el test de correlación de Pearson, de manera on-line en el enlace del siguiente dominio web: (https://www.socscistatistics.com/tests/pearson/default2.aspx.) para examinar la comparación de las emociones con la calificación que le arroga el tutor virtual al sujeto luego de la interacción. Para ello se utilizó los datos en porcentajes de este modo el análisis no se vea afectado por diferencias de tiempo en las sesiones. Luego en la Tabla 6 se observan los datos obtenidos en el test de correlación de Pearson.

\section{Tabla 6}

Resultados Test de Pearson

\begin{tabular}{lc}
\hline Emoción & Valor de R \\
\hline Ira & 0,0362 \\
Desprecio & $-0,0645$ \\
Asco & 0,2425 \\
Miedo & $-0,5601$ \\
Felicidad & 0,4738 \\
Neutral & $-0,7395$ \\
Tristeza & $-0,0814$ \\
Sorpresa & 0,3254 \\
\hline
\end{tabular}


Con respecto a la discusión o análisis de los videos algunos sujetos no reflejan en las emociones un valor numérico en otras palabras (0), tal como se observa en la Tabla 3 las razones pueden ser las siguientes: es posible que la cámara no estaba bien situada y por tanto no se grabó bien el rostro completo del sujeto, también puede ser que el sujeto se esté tapando demasiado la cara ya sea con otra parte del cuerpo o con alguna ropa, en ambos casos es un fallo del procedimiento. Además, es posible que el sujeto estaba desinteresado con la sesión de aprendizaje mediante el tutor virtual y quite su atención de la pantalla del ordenador, por ejemplo, si el usuario mira el móvil, mira hacia abajo o para otro lado, en estos casos no aporta ningún tipo de información útil al experimento.

En igual forma los resultados de la Tabla 4 de los datos en porcentajes muestra la emoción neutral en todos los sujetos analizados en más del 95\% del tiempo mientras interactúan con el tutor virtual, esto es evidente tratándose de un proceso de aprendizaje. Las emociones que en la mayoría de los sujetos están en $0 \%$ del tiempo como ira, asco, miedo y sorpresa, basta con que se modifiquen un poco para que aporten mucha información al experimento. Por lo tanto, son variables importantes a la hora del análisis y no suelen darse con frecuencia.

En otro sentido la emoción que cambia mucho su valor de un sujeto a otro como tristeza proporciona una valiosa información, esta emoción depende en gran medida del sujeto que se esté analizando. Por último, hay otras emociones como felicidad y desprecio que muestran valores muy bajos, aunque no sean exactamente $0 \%$ y en algunas ocasiones alcanzan picos de hasta $19 \%$. En efecto son emociones que se expresan durante el proceso de aprendizaje, pero con poca frecuencia.

Una vez analizados los resultados del experimento de forma global se examinaron las notas que ha atribuido el tutor virtual al sujeto como se observan en la Tabla 5. Existen todo tipo de notas incluso sujetos que se le ha ubicado una nota perteneciente a los extremos. En todo caso el balance de las calificaciones es muy positivo lo que provocó unas posteriores conclusiones que están a favor de este sistema de aprendizaje.

El análisis procedente de los resultados del test de correlación de Pearson que se visualizan en la Tabla 6 evidencia que el valor de R va desde 1 a -1 , significando ambos extremos un alto nivel de correlación. La escala de interpretación del coeficiente de correlación de Pearson se muestra en la Tabla 1.

En función de lo planteado en la explicación anterior se puede decir que ninguna de las emociones alcanza una fuerte correlación, aunque algunas están más asociadas que otras ya sean de forma positiva o negativa. Las variables que están menos correlacionadas hasta el punto que la relación es casi nula, es decir que no influyen en la nota del sujeto son: 
- Ira. - Esta emoción a pesar de ser positiva alcanza una relación entre sus variables muy débil por mostrarse pocas veces en los sujetos, puesto que es muy difícil que se exprese la emoción durante una sesión de aprendizaje.

- Desprecio. - Aunque técnicamente es una emoción negativa la correlación es muy baja, se manifiesta mientras que un sujeto intenta comprender conocimientos, sobre todo si son nuevos o complejos.

- Tristeza. - Se correlaciona negativamente con la nota del tutor virtual, puesto que es probable que se manifieste pocas veces en los sujetos durante su interacción y suceda por la desgana del sujeto o no está feliz ante la realización de la sesión.

El resto de emociones están más correlacionadas con la nota que el tutor virtual ha atribuido al sujeto después de la interacción que las listadas anteriormente. Cabe mencionar que esta relación no es muy fuerte puesto que no llega a 0,7 en la mayoría de los casos. Estas variables son: felicidad, sorpresa, asco, miedo y neutral. Las emociones que están relacionadas de forma positiva con la nota del tutor virtual son: felicidad, sorpresa y asco. Por otra parte, las emociones que están relacionadas de forma negativa son miedo y neutral.

A continuación, se detallan los motivos del porque estas emociones están correlacionadas con la nota del sujeto.

- Felicidad. - Esta emoción alcanza una correlación positiva moderada debido a que aparece algunas veces en los sujetos en la interacción de aprendizaje, es probable que el sujeto se sienta aceptado, valorado, respetado e inclusive ayudado por sus compañeros o tutor en un momento puntual o determinado.

- Sorpresa. - La emoción tiene una correlación positiva baja con la nota del tutor virtual, es decir cuando el sujeto entiende los conocimientos mientras interactúa o cuando sabe con seguridad la respuesta a una pregunta puede llegar a mostrar esta emoción. Evidentemente cuanto más se manifiesta esta emoción significa que el sujeto entendió los conocimientos y por lo tanto se refleja positivamente en la nota.

- Asco. - Técnicamente es una correlación positiva baja al igual que la anterior. Cuando más cercano está al valor del cero más débil es la relación. En efecto pocas veces un sujeto en una sesión de aprendizaje muestra la emoción asco, a no ser que se sintió ofendido, desagradable o se disgustó con alguna persona en un momento puntual.

- Miedo. - Alcanzó una relación negativa moderada lo que representa una aproximación a -1. Las razones se deben cuando un sujeto está sometiendo a una sesión de aprendizaje y no está seguro de lo que aprende o a la hora de responder a la solución de una pregunta puede llegar a mostrar la emoción miedo.

- Neutral. - Es la variable que alcanza la correlación más alta de todas, esta correlación evidentemente es negativa alta. Considerando que en la mayoría de vídeos analizados 
la mayor parte del tiempo el sujeto está neutral, es decir es lo "normal" mientras que un alumno está atendiendo y/o realizando ejercicios.

Posteriormente del análisis de los resultados, se obtendrán las conclusiones que responderán a las preguntas iniciales y que servirán para el cumplimiento del proceso de experimentación.

\section{Conclusiones}

- En términos generales, éste trabajo se orientó en el análisis del comportamiento emocional en estudiantes de educación secundaria y la relación con el aprendizaje virtual. A través de los experimentos ejecutados se hace referencia a los fotogramas no analizados, tras la reflexión realizada sobre los mismos anteriormente la opción más probable para que un fotograma se clasifique como "no analizado" es la falta de atención del alumno y que por lo tanto se podría considerar como una valiosa información, sin embargo ante la existencia del otro caso explicado anteriormente, es decir que sea producto de un fallo humano (como la colocación de la cámara), los fotogramas clasificados como "no analizados" no podrán llegar a ser fotogramas útiles.

- Después de realizar el test de correlación de Pearson se demuestra que las variables no están fuertemente correlacionadas con la nota que atribuye el tutor virtual al sujeto después de la interacción, no obstante, sí influyen en la misma. Esto se debe a que todas las personas son distintas entonces, aunque encontremos algunas similitudes en este sentido demostradas por profesionales en la materia como profesores o psicólogos no quiere decir que se cumplan sí o sí en todas las personas. Estas semejanzas mencionadas anteriormente pueden ser, si un alumno muestra tristeza significa que no le gusta lo que está aprendiendo y por tanto aprenderá menos que otro alumno que no esté triste o si un alumno se muestra concentrado adquirirá más conocimientos que uno que no lo está.

- En definitiva, el producto del proceso experimental realizado llega a la conclusión de que los sistemas de aprendizaje con un tutor virtual son buenos para el alumnado. Todo esto se puede demostrar gracias a los análisis de los vídeos de los sujetos que han participado en el proyecto. Estos resultados se pueden extrapolar a toda la población, aunque una de las limitaciones del proyecto fue debido a la pandemia que se atraviesa no se pudo aplicar a toda la muestra estratificada que se había planificado en un principio y se abordó solo a un cierto grupo de estudiantes que contaban con las herramientas y medios tecnológicos adecuados. De tal forma se puede indicar que se debe continuar mejorando en este tipo de enseñanza utilizando las TIC de forma más concreta y así pasar de una metodología de aprendizaje clásica a otro tipo de metodología que implique las TIC como factor clave. 
- Como trabajo futuro se propone implementar análisis en paralelo en la aplicación de forma que el usuario tenga la posibilidad de analizar varios vídeos a la vez, de esta manera el usuario escoja en cada uno de los análisis paralelos la suscripción de Api Face que desea utilizar, aunque si cuenta con una versión de pago podrá analizar con esa cuenta todos los vídeos que desee de forma paralela. Esto supondrá una mayor rapidez de análisis y por tanto una ventaja para el usuario. Además, permitir al usuario almacenar los resultados en la nube, esta opción puede ser muy interesante si se decide analizar un vídeo de varias horas de duración y el usuario desea conservar cada uno de los fotogramas extraídos.

\section{Referencias bibliográficas}

Ángel, J., \& Retana, G. (2012). La educación emocional, su importancia en el proceso de aprendizaje. Revista Educacion, 36(1), 1-24.

Awofala, A. O., Olabiyi, O. S., Awofala, A. A., Arigbabu, A. A., Fatade, A. O., \& Udeani, U. N. (2019). Attitudes toward Computer, Computer Anxiety and Gender as determinants of Pre-service Science, Technology and Mathematics Teachers' Computer Self-efficacy. Digital Education Review, (36), 51-67.

Barrera Erreyes, H. M., Barragán García, T. M., \& Ortega Zurita, G. E. (2017). La realidad educativa ecuatoriana. Revista Iberoamericana de Educación, 75, 9-20.

Bisandu, D. B. (2016). Design Science Research Methodology in Computer Science and Information Systems. International Journal of Information Technology, (November 2016), 1-7.

Bledsoe, W. W. (1963). Informe de un proyecto de reconocimiento facial. February 23, 2020, https://archive.org/details/firstfacialrecognitionresearch/page/n3/mode/2up

Bogarín, A., Cerezo, R., \& Romero, C. (2017). A survey on educational process mining. 17

Carretero Bermejo, R. (2015). Intelingencia emocional y sexismo: estudio del componente del emocional sexismo (UNED). https://ebookcentral.proquest.com/lib/uteclvtsp/ reader.action?docID $=4775759 \&$ query $=$ Goleman $+\% 281995 \% 29$

Cerezo, R., Bogarín, A., Esteban, M., \& Romero, C. (2020). Process mining for selfregulated learning assessment in e-learning. Journal of Computing in Higher Education, 32(1), 74-88.

Chango, W., Cerezo, R., \& Romero, C. (2019). Predicting academic performance of 
university students from multi-sources data in blended learning. ACM International Conference Proceeding Series. Association for Computing Machinery.

Cifuentes, M. (2013). La influencia de la inteligencia emocional en el rendimiento matemático de alumnos de educación secundaria. Aplicación de un programa de intervención psicopedagógica de educación emocional. 388. https://repositorio.ucjc.edu/.../Tesis Doctoral_M ${ }^{\mathrm{a}}$ Elisabet

Clarizia, F., Colace, F., De Santo, M., Lombardi, M., Pascale, F., \& Pietrosanto, A. (2018). E-learning and sentiment analysis: A case study. ACM International Conference Proceeding Series, 111-118.

Cordero, J., \& Aguilar, J. (2016). Reconocimiento multimodal de emociones humanas en un ambiente inteligente. (September), 107.

Dresch, A., Pacheco Lacersa, D., \& Valle Antunes, A. (2018). Design-science research, A Method for Science and Technology Advancement. In Research Methods: Information, Systems, and Contexts: Second Edition.

Engeness, I., Nohr, M., Singh, A. B., \& Mørch, A. (2019). Use of videos in the Information and Communication Technology Massive Open Online Course: Insights for learning and development of transformative digital agency with pre- and in-service teachers in Norway. Policy Futures in Education, 1-20.

Espinoza Freire, E. E., \& Ricaldi Echevarríaz, M. L. (2018). El tutor en los entornos virtuales de aprendizaje. Universidad y Sociedad, 10(3), 201-210.

Estrada Villa, E. J., \& Boude Figueredo, O. R. (2015). Hacia una Propuesta Para Evaluar Ambientes Virtuales de Aprendizaje en Educación Superior. Academia y Virtualidad, 8(2), 14.

Franco Moreno, Y. M. (2017). Rol del Tutor en el Contexto del Aprendizaje Virtual. Journal of Chemical Information and Modeling, 2(6), 270-285.

Greene, J. A., \& Azevedo, R. (2010). The measurement of learners' self-regulated cognitive and metacognitive processes while using computer-based learning environments. Educational Psychologist, 45(4), 203-209.

Harley, J. M., Bouchet, F., Hussain, M. S., Azevedo, R., \& Calvo, R. (2015). A multicomponential analysis of emotions during complex learning with an intelligent multi-agent system. Computers in Human Behavior, 48, 615-625.

Hernández Lalinde, J., Espinosa Castro, J., Peñaloza Tarazona, M., Fernández González, J., Chacón Rangel, J., Toloza Sierra, C., ... Bermúdez Pirela, V. (2018). Sobre El 
Uso Adecuado Del Coeficiente De Correlación De Pearson: Definición, Propiedades Y Suposiciones. AVFT - Archivos Venezolanos de Farmacología y Terapéutica, $37(5)$.

Hernandez Sampieri, R., Fernandez Collado, C., \& Baptista Lucio, M. del P. (2010). Metodología de la investigación. In J. Mares Chacón (Ed.), Metodología de la investigación (Quinta Ed.). http://www.casadellibro.com/libro-metodologia-de-lainvestigacion-5-ed-incluye-cd-rom/9786071502919/1960006

Hew, K. F., \& Brush, T. (2007). Integrating technology into K-12 teaching and learning: Current knowledge gaps and recommendations for future research. Educational Technology Research and Development, 55(3), 223-252.

Hew, K. F., \& Cheung, W. S. (2014). Students' and instructors' use of massive open online courses (MOOCs): Motivations and challenges. Educational Research Review, 12, 45-58.

Khalfallah, J., \& Ben Hadj Slama, J. (2019). The effect of emotional analysis on the improvement of experimental e-learning systems. Computer Applications in Engineering Education, 27(2), 303-318.

Kumar, J. A., Muniandy, B., \& Wan Yahaya, W. A. J. (2019). Exploring the effects of emotional design and emotional intelligence in multimedia-based learning: an engineering educational perspective. New Review of Hypermedia and Multimedia, 25(1-2), 57-86.

León Porras, C. J. P. (2009). La inteligencia emocional en estudiantes de educación superior universitaria y no universitaria de Administración de Empresas. 16. https://ebookcentral.proquest.com/lib/uteclvtsp/detail.action?docID=3183493

López Munguía, O. (2008). La Inteligencia emocional y las estrategias de aprendizaje como predictores del rendimiento académico en estudiantes universitarios. http://cybertesis.unmsm.edu.pe/handle/cybertesis/615

Madrid Tamayo, T. (2019). El sistema educativo de Ecuador: un sistema, dos mundos. Revista Andina de Educación, 2(1), 8-17.

Mao, Q. R., Pan, X. Y., Zhan, Y. Z., \& Shen, X. J. (2015). Using Kinect for real-time emotion recognition via facial expressions. Frontiers of Information Technology and Electronic Engineering, 16(4), 272-282.

Mega, C., Ronconi, L., \& De Beni, R. (2014). What makes a good student? How emotions, self-regulated learning, and motivation contribute to academic Achievement. Journal of Educational Psychology, 106(1), 121-131. 
Molinari, G., Trannois, M., Tabard, A., \& Lavoué, E. (2016). EMORE-L: An emotion reporting tool for distance learning. Actes de La 28ième Conférence Francophone Sur l'Interaction Homme-Machine, 167-176.

Asamblea Nacional. (2015). Reglamento General a la ley orgánica de educación intercultural. Registro Oficial, (4), 3-7.

Nikopoulou, R., Spyrou, E., Vernikos, I., \& Mylonas, P. (2018). Emotion recognition from speech: A classroom experiment. ACM International Conference Proceeding Series, 104-105.

Picciano, A. G. (2013). Theories and Frameworks for Online Education : Seeking an Integrated Model. 166-190.

Ramachandiran, C. R., Mahmud, M. M., \& Jomhari, N. (2019). A revolutionary approach in virtual learning: Usercentered kansei virtual agent. ACM International Conference Proceeding Series, 7-11.

Salazar P, C., \& Castillo G, S. (2018). Fundamentos básicos de Estadística (Primera Ed). Quito.

Saneiro, M., Santos, O., Salmeron-Majadas, S., \& Boticario, J. (2015). Towards using influence diagram on social-network based analysis for managing students' collaborations. Computer-Supported Collaborative Learning Conference, CSCL, 2, $647-648$.

Sautu, R. (2005). TODO es TEORÍA Objetivos y métodos de investigación (Lumiere). Buenos Aires.

Taber, K. S. (2018). The Use of Cronbach's Alpha When Developing and Reporting Research Instruments in Science Education. Research in Science Education, 48(6), 1273-1296.

Taruchaín Pozo, L. F., Freire Palacios, V. A., \& Mayorga Álvarez, M. de los Á. (2020). Bienestar psicológico y estrés laboral en trabajadores no profesionales de la ciudad de Ambato, aplicando el cuestionario de salud general de Goldberg GHQ-12. Ciencia Digital, 4(1.1.), 32-44.

Zhong, S. H., Li, Y., Liu, Y., \& Wang, Z. (2017). A computational investigation of learning behaviors in MOOCs. Computer Applications in Engineering Education, (March), 1-13.

Zimmerman, B. (1990). Self-Regulated Learning and Academic Achievement: An Overview. Educational Psychologist, 25(1), 3-17. 


\section{Conciencia}

El artículo que se publica es de exclusiva responsabilidad de los autores y no necesariamente reflejan el pensamiento de la Revista Conciencia Digital.

\section{Ciencia}

El artículo queda en propiedad de la revista y, por tanto, su publicación parcial y/o total en otro medio tiene que ser autorizado por el director de la Revista Conciencia Digital.
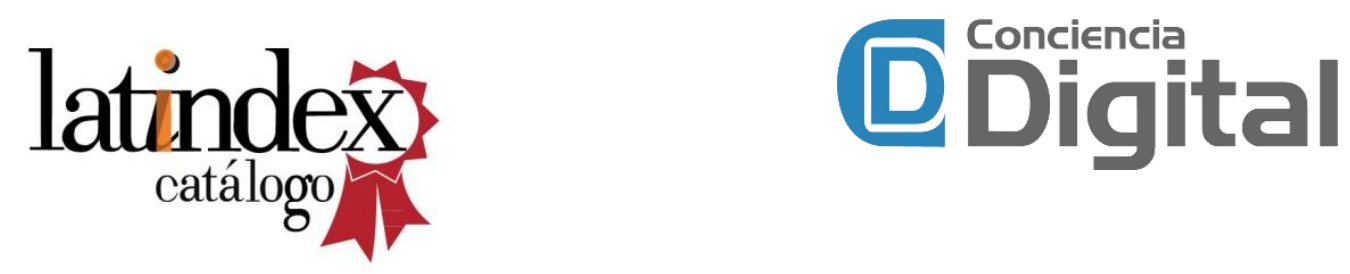

Indexaciones

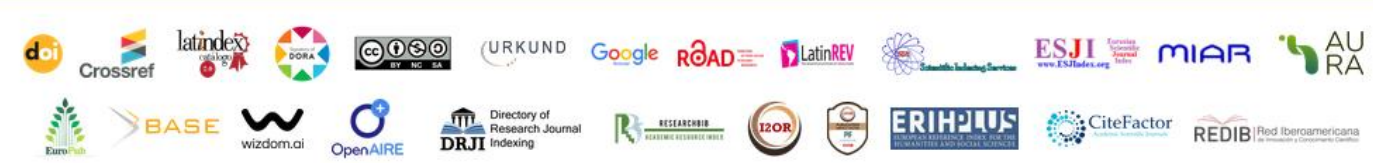

